\title{
Artigos
}

\section{Déficit de autocuidado em idosos: características, fatores associados e recomendações às equipes de Estratégia Saúde da Família}

Self-care deficit in the elderly: characteristics, associated factors and recommendations to Family Health Strategy teams (abstract: p. 15)

Déficit de autocuidado en ancianos: características, factores asociados y recomendaciones a los equipos de Estrategia Salud de la Familia (resumen: p. 15)

\section{Lúcia Soares Buss Coutinho(a)}

$<$ luciacoutinho@uniplaclages.edu.br>

Elaine Tomasi ${ }^{(b)}$

<elaine.tomasi@ufpel.edu.br> (a) Curso de Medicina, Universidade do Planalto Catarinense. Avenida Mal. Castelo Branco, 170, Bairro Universitário. Lages, SC, Brasil. 88509-900.

(b) Departamento de Medicina Social, Faculdade de Medicina, Universidade Federal de Pelotas. Pelotas, RS, Brasil.

O objetivo deste trabalho foi caracterizar o déficit de autocuidado em associação com características sociodemográficas, comportamentais, de condição de saúde, de acesso e utilização de serviços de saúde. Realizou-se um inquérito populacional e transversal com idosos adscritos ao território de equipe de Saúde da Família, em Lages, SC, Brasil. O déficit de autocuidado foi aferido com Appraisal of Self-Care Agency Scale (ASA-A). Entre os entrevistados, a maioria era do sexo feminino, branca, com idade entre sessenta e setenta anos. A prevalência do desfecho foi de $25 \%$ e relacionou-se significativamente a multimorbidade, pior autopercepção de saúde, consumo alimentar inadequado, baixa escolaridade, inatividade e dependência para as atividades básicas da vida diária, reforçando o papel das equipes de saúde em promover iniciativas de autocuidado, aproximando o cidadão de políticas públicas e recursos da comunidade local.

Palavras-chave: Autocuidado. Envelhecimento. Multimorbidade. Atenção Primária à Saúde. Estratégia Saúde da Família.

Coutinho LSB, Tomasi E. Déficit de autocuidado em idosos: características, fatores associados e recomendações às equipes de Estratégia Saúde da Família. Interface (Botucatu). 2020; 24(Supl. 1): e190578 https://doi.org/10.1590/Interface.190578 


\section{Introdução}

Em todo o mundo, um dos aspectos mais relacionados ao fenômeno da transição epidemiológica é o crescimento da população idosa ${ }^{1}$. A cada ano, 650 mil novos idosos são incorporados à população brasileira ${ }^{2}$, em um contexto nacional de acentuada desigualdade social, pobreza e fragilidade das instituiçốes de saúde ${ }^{3-5}$.

A carga global de doenças não transmissíveis (DNT) e a multimorbidade, características dessa população, mostram-se como um problema de saúde pública de grande relevância, uma vez que atinge mais de 50\% dos idosos no Brasil e reflete no maior risco de morte, no declínio funcional do idoso e no impacto negativo sobre sua qualidade de vida ${ }^{3,4,6}$.

Observa-se uma crise contemporânea dos sistemas de atenção à saúde decorrente da incoerência entre a situação de transição demográfica e o modo como se estruturam os serviços; afinal, não se pode continuar a ofertar cuidados de saúde voltados prioritariamente para as condições agudas ou para as agudizações das condições crônicas, organizados de forma fragmentada ${ }^{7,8}$.

Nesse contexto, surgem as Redes de Atenção à Saúde (RAS), que buscam dar respostas de qualidade, eficientes e efetivas no enfrentamento da tripla carga de doenças. A meta é organizar os serviços de saúde de forma a permitir uma atenção contínua e integral, por meio de cuidados continuados prestados pelos diferentes serviços de saúde, de uma forma articulada entre si e coordenados pela Atenção Primária à Saúde (APS) ${ }^{6,9}$.

Ao mesmo tempo, consolida-se o Modelo de Atenção às Condições Crônicas (MACC), propondo mudanças importantes no desenho do sistema de prestação de serviços, enfatizando o autocuidado apoiado e iniciando um movimento de corresponsabilização das pessoas pela sua condição de saúde $e^{7,8}$.

A Estratégia Saúde da Família (ESF) passa a se destacar como locus privilegiado para o cuidado dos idosos e de suas multimorbidades, singularizando os cuidados de saúde; centralizando a pessoa, e não mais na doença $a^{10-12}$; e buscando um equilíbrio entre a atenção à demanda espontânea e a atenção continuada ${ }^{9}$. Além disso, a ênfase no trabalho em equipe multidisciplinar, apoiado em ferramentas de tomada de decisão, procura garantir o princípio da equidade à luz dos determinantes sociais da saúde, considerando o meio em que vivem os indivíduos e reconhecendo seus saberes para o autocuidado-12.

Por outro lado, segundo as Diretrizes para o Cuidado das Pessoas com Doenças Crônicas nas Redes de Atenção à Saúde, instrumentalizar o autocuidado é muito mais do que dizer às pessoas o que devem fazer. $\mathrm{O}$ objetivo do autocuidado apoiado implica em cooperação entre a equipe de saúde e os usuários para, conjuntamente, definir os problemas, estabelecer as metas, monitorá-las, instituir os planos de cuidado e resolver as intercorrências ${ }^{13}$.

Dessa forma, importa compreender que o entendimento sobre o autocuidado se construiu ao longo do tempo em resposta ao movimento de unilateralização na tomada de decisão e quase ausência da participação dos indivíduos sobre as escolhas em relação à saúde, fruto de um momento de grande avanço das tecnologias duras e profissionalização do cuidado médico ${ }^{14 \cdot 16}$. 
Conceitualmente, o autocuidado evolui desde o conjunto de açóes que o indivíduo desenvolve consciente e deliberadamente em seu benefício no sentido de manter a vida e o seu bem-estar ${ }^{15-17}$ até uma construção coletiva que aponta não só para a responsabilidade do indivíduo, mas também considera o apoio da família, dos amigos e das instituiçôes de saúde como essenciais na contribuição para que os indivíduos tomem as melhores decisóes para o seu bem-estar ${ }^{18}$.

Já a capacidade de autocuidado é definida como aquilo que a pessoa é capaz de realizar por si e para si própria, com base em três elementos básicos:

1. Disposiçóes e capacidades fundamentais, relacionadas à condição de saúde dos indivíduos e à sua capacidade para executar as atividades básicas ou instrumentais da vida diária.

2. Componentes de poder, ligados aos aprendizados específicos e à compreensão da necessidade de mudar as coisas.

3. Operaçóes de autocuidado propriamente ditas, elemento que amplia o conceito de autocuidado demonstrando que é necessário mais do que ter as condiçôes fundamentais e o conhecimento para desenvolvê-lo, ou seja, que é necessário também decidir o que fazer e quando agir para alcançar a mudança ou atingir a regulação $\mathrm{o}^{15-17}$.

Assim, o objetivo deste trabalho foi caracterizar os idosos com déficit de autocuidado do território investigando sua associação com as características sociodemográficas; comportamentais; de condição de saúde; e de acesso e utilização de serviços de saúde. Entende-se que reunir dados sobre o autocuidado da população local - para servir de subsídio a um programa de educação permanente direcionado aos profissionais da ESF, abrindo espaço para melhor compreender o território do ponto de vista das capacidades de autocuidado dos indivíduos; e discutir e aperfeiçoar os processos de trabalho da equipe, fortalecendo o compromisso de todos com a promoção do autocuidado e com um processo de envelhecimento saudável - esteja alinhado à Política Nacional de Educação Permanente em Saúde ${ }^{19}$.

\section{Métodos}

Foi realizado um inquérito populacional de delineamento transversal, em uma amostra de pessoas com sessenta anos ou mais, adscritas ao território de abrangência de uma equipe de Saúde da Família (SF), na área urbana de município de porte médio no sul do Brasil.

Inicialmente, foi concluído um levantamento populacional para identificar todas as pessoas moradoras da área de abrangência da equipe de SF 44, incluindo aquelas sem cadastro formal no serviço. De acordo com as informaçóes coletadas pelos agentes comunitários de saúde (ACS), o total de residentes na época era de 3.179 pessoas, das quais 552 eram idosos $(17,4 \%)$.

A seguir, todos os domicílios onde residiam pessoas idosas foram mapeados, optando-se por limitar a amostra a um entrevistado por domicílio, previamente escolhido, de forma aleatória, totalizando 419 idosos. 
Foram excluídos do estudo aqueles idosos que se mudaram para além dos limites do território ou aqueles que faleceram, no período de tempo entre o levantamento populacional e o início do trabalho de campo, além daqueles portadores de alguma deficiência física ou mental incapazes de responder ao instrumento. Os idosos que não estiveram presentes em nenhuma das três tentativas de visita foram considerados perdas e aqueles que se negaram a responder o questionário tiveram as recusas contabilizadas.

A coleta de dados ocorreu entre setembro e dezembro de 2018, por meio de questionário padronizado e pré-codificado, aplicado no próprio domicílio. $\mathrm{O}$ trabalho de campo envolveu a seleção e capacitação de 17 entrevistadores voluntários, sendo nove profissionais da equipe de SF e os demais, estudantes de Medicina da Universidade do Planalto Catarinense (Uniplac), inseridos no território por meio de atividades de integração ensino-serviço.

Foi realizado um estudo-piloto com vinte idosos moradores de outras áreas de abrangência da Unidade de Saúde da Família (USF) Coral, buscando preparar os entrevistadores, qualificar o instrumento e quantificar o tempo médio da coleta de dados. Além disso, o contato próximo com a equipe para recebimento e verificação dos instrumentos, bem como o esclarecimentos de dúvidas, sempre foi prioridade.

O desfecho do estudo foi o déficit de autocuidado, aferido por meio do instrumento Appraisal of Self-Care Agency Scale (ASA-A) anteriormente traduzido, validado e adaptado culturalmente para idosos no Brasil, atingindo bom desempenho psicométrico, alta consistência interna e estabilidade ou teste-reteste, assim como validade discriminante e concorrente ${ }^{17}$.

O instrumento se refere à disponibilidade, à vontade e às condiçóes das pessoas para modificar suas vidas, melhorando-as. Também avalia os cuidados com alimentação, higiene e peso. Os itens aferem ainda se os respondentes procuram realizar adaptaçóes para melhorar a própria saúde e se buscam rede de apoio em caso de dificuldades com os procedimentos de autocuidado ${ }^{17}$.

A variável de desfecho foi obtida inicialmente por meio do somatório da pontuação de cada um dos 24 itens da escala, de acordo com suas opçóes de resposta - discordo totalmente (1 ponto); discordo (2 pontos); nem concordo, nem discordo (3 pontos); concordo ( 4 pontos); e concordo totalmente $(5 \text { pontos })^{17}$-, sendo recodificada, a seguir, de acordo com os quartis de sua distribuição. Assim, os idosos classificados no quartil inferior foram considerados com déficit de autocuidado, evidenciando-se aqueles indivíduos do território sob maior risco em virtude de suas menores capacidades para o autocuidado.

As variáveis independentes sociodemográficas foram: sexo (masculino ou feminino), idade em anos completos (sessenta a 64, 65 a setenta, 71 a 79 ou oitenta a 96), cor da pele autorreferida (parda/morena/preta ou branca), ter companheiro (sim ou não), escolaridade (superior, médio, fundamental completo ou fundamental incompleto), quartis de renda per capita em reais (143 a 750, 751 a mil, 1001 a 1750, ou1751 a sete mil), ocupação (não aposentado e trabalha, aposentado e não trabalha, aposentado e trabalha ou não aposentado e não trabalha) e recebe algum benefício (sim ou não). 
Os fatores comportamentais investigados foram: fumo ${ }^{20}$ (nunca fumou, ex-fumante ou fumante); abuso de álcool, segundo Vigitel ${ }^{21}$ (sim ou não); cuida de algum familiar (sim ou não); atividades básicas de vida, segundo escala de Katz ${ }^{22}$ (dependente ou independente); atividades instrumentais de vida diária, segundo Lawton ${ }^{23}$ (dependente ou independente); funcionalidade familiar ${ }^{24}$ (boa funcionalidade, moderada disfunção ou elevada disfunção); atividade física, segundo $\operatorname{Ipaq}^{25}$ (ativo ou inativo); consumo regular de frutas e hortaliças ${ }^{21}$ (sim ou não), feijão $0^{21}$ (sim ou não), carne com excesso de gordura $^{21}$ (sim ou não), leite integral ${ }^{21}$ (sim ou não), doces $^{21}$ (sim ou não) e refrigerantes ${ }^{21}$ ( iim ou não); e substituição do almoço ou janta por lanches ${ }^{21}$ (sim ou não).

As variáveis que abordaram as características de saúde e acesso/utilização de serviços de saúde foram: autopercepção de saúde ${ }^{26}$ (muito boa, boa, regular, ruim ou muito ruim); multimorbidade ${ }^{27}$ (sim ou não); necessidade de utilização do serviço de saúde (sim ou não); se conseguiu ser atendido (sim ou não); se utiliza a USF Coral (sim ou não); e orientações recebidas dos profissionais de saúde sobre alimentação saudável (sim ou não), saúde bucal (sim ou não), risco de quedas (sim ou não), atividade física (sim ou não), uso correto da medicação (sim ou não) e acolhimento e classificação de risco (sim ou não).

Os questionários foram codificados, revisados e submetidos à dupla digitação por meio do aplicativo EpiData ${ }^{\circledR}$ versão 3.128. O banco de dados final foi exportado por meio do aplicativo Stat Transfer 5.0 para o pacote estatístico Stata 15.0, no qual foram realizadas as análises por meio do teste do qui-quadrado, razóes de prevalência e intervalos de confiança de $95 \%$.

O estudo contou com carta de anuência da Secretaria Municipal de Saúde de Lages e foi aprovado pelo Comitê de Ética em Pesquisa da Faculdade de Medicina da Universidade Federal de Pelotas (UFPEL) conforme parecer n. 2.782.544. A todos os entrevistados foi garantido o direito de não participação e o sigilo em relação à sua identificação diante das informaçóes prestadas. Aos que concordaram em responder o questionário, foi solicitada assinatura de Termo de Consentimento Livre e Esclarecido.

\section{Resultados}

Após estimar o quantitativo de idosos no território $(\mathrm{n}=552)$, houve uma delimitação da população-alvo a um idoso por domicílio, havendo uma redução do número de idosos elegíveis $(n=419)$. Em virtude do intervalo de tempo entre o levantamento populacional e o início do trabalho de campo, acabou-se por excluir 34 idosos, sendo 12 destes por óbito e 22 por motivo de mudança de residência para fora dos limites do território antes do início das entrevistas $(\mathrm{n}=385)$. Por fim, foram excluídos outros oito indivíduos portadores de alguma deficiência física ou mental incapazes de responder ao instrumento $(\mathrm{n}=377)$, houve 33 perdas e foram registradas seis recusas, chegando-se ao total de 338 entrevistados, que correspondem a $90 \%$ da amostra.

A maioria dos entrevistados era do sexo feminino (65\%) e 54\% tinham entre 60 e 70 anos. A cor da pele branca foi referida por $86 \%$ e praticamente metade dos indivíduos (52\%) viviam com companheiro(a). Dois quintos dos entrevistados não tinham ensino fundamental completo e $67 \%$ referiram estar aposentado e não trabalhar. Para metade da amostra, a renda familiar per capita oscilou entre $\mathrm{R} \$ 751,00$ e $\mathrm{R} \$ 1.750,00$ mensais (tabela 1). 
Tabela 1. Distribuição da amostra e déficit de autocuidado de acordo com características sociodemográficas. USF Coral, Lages, SC, 2018.

\begin{tabular}{|c|c|c|c|c|}
\hline Característica & $\mathrm{n}(\%)$ & $\begin{array}{c}\text { Déficit de } \\
\text { autocuidado }\end{array}$ & Razão de prevalências (IC95\%) & p-valor \\
\hline Sexo & & & & 0,327 \\
\hline Masculino & $117(34,6 \%)$ & $29,1 \%$ & 1,00 & \\
\hline Feminino & $221(65,4 \%)$ & $23,5 \%$ & $0,81(0,85-1,79)$ & \\
\hline Idade (anos) & & & & 0,448 \\
\hline De 60 a 64 & $89(26,3 \%)$ & $24,7 \%$ & 1,00 & \\
\hline De 65 a 70 & $93(27,5 \%)$ & $21,5 \%$ & $0,87(0,51-1,48)$ & \\
\hline De 71 a 79 & $106(31,4 \%)$ & $28,3 \%$ & $1,15(0,72-1,84)$ & \\
\hline De 80 a 96 & $50(14,8 \%)$ & $28,0 \%$ & $1,13(0,64-2,01)$ & \\
\hline Cor da pele & & & & 0,991 \\
\hline Parda/morena/preta & $49(14,5 \%)$ & $26,5 \%$ & 1,00 & \\
\hline Branca & $289(85,5 \%)$ & $25,3 \%$ & $0,95(0,57-1,58)$ & \\
\hline Tem companheiro & & & & 0,883 \\
\hline Não & $161(47,8 \%)$ & $24,8 \%$ & 1,00 & \\
\hline Sim & $176(52,2 \%)$ & $26,1 \%$ & $1,05(0,73-1,52)$ & \\
\hline Escolaridade & & & & 0,026 \\
\hline Superior & $47(14,7 \%)$ & $19,1 \%$ & 1,00 & \\
\hline Médio & $67(21,0 \%)$ & $19,4 \%$ & $1,01(0,47-2,17)$ & \\
\hline Fundamental completo & $75(23,5 \%)$ & $25,3 \%$ & $1,32(0,65-2,68)$ & \\
\hline Fundamental incompleto & $130(40,8 \%)$ & $32,3 \%$ & $1,69(0,89-3,19)$ & \\
\hline Quartis de renda per capita (reais) & & & & 0,073 \\
\hline De 1.751 a 7.000 & $78(23,9 \%)$ & $23,1 \%$ & 1,00 & \\
\hline De 1.001 a 1.750 & $76(23,2 \%)$ & $17,1 \%$ & $0,74(0,39-1,41)$ & \\
\hline De 751 a 1.000 & $87(26,6 \%)$ & $31,0 \%$ & $1,35(0,81-2,24)$ & \\
\hline De 143 a 750 & $86(26,3 \%)$ & $31,4 \%$ & $1,36(0,82-2,27)$ & \\
\hline Aposentadoria e trabalho & & & & 0,762 \\
\hline Não aposentado e trabalha & $19(5,8 \%)$ & $15,8 \%$ & 1,00 & \\
\hline Aposentado e não trabalha & $218(66,5 \%)$ & $24,8 \%$ & $1,57(0,54-4,55)$ & \\
\hline Aposentado e trabalha & $51(15,5 \%)$ & $27,5 \%$ & $1,74(0,56-5,38)$ & \\
\hline Não aposentado e não trabalha & $40(12,2 \%)$ & $27,5 \%$ & $1,74(0,55-5,22)$ & \\
\hline
\end{tabular}

Quanto ao consumo de álcool e tabaco, $14 \%$ dos idosos eram fumantes e o abuso de álcool foi registrado para $16 \%$ dos homens e $3 \%$ das mulheres (IC 95\% 1,28-5,10). Já a dependência para atividades básicas da vida diária atingiu $8 \%$ da amostra e a dependência para atividades instrumentais chegou a $18 \%$ entre os homens e $29 \%$ entre as mulheres.

Mais de metade dos idosos foram classificados como inativos, $15 \%$ referiram ser responsáveis pelo cuidado de algum familiar e $11 \%$ apresentavam algum grau de disfunção familiar. Cerca de dois terços dos entrevistados referiram consumir regularmente frutas, hortaliças e leite integral. O consumo regular de feijão foi referido por $52 \%$ e o de doces, por $28 \%$ dos idosos. Consumir regularmente carne com gordura e substituir refeiçôes por lanches foi citado por $22 \%$ e $7 \%$ referiu consumo regular de refrigerantes (tabela 1 ).

Metade dos idosos (51\%) percebia sua saúde como boa ou muito boa e cerca de três quartos referiu cinco ou mais problemas de saúde. Precisaram ser atendidos em serviços de saúde $75 \%$ dos entrevistados e, destes, apenas três não conseguiram ser atendidos. 
Entre os entrevistados, 278 idosos (82\%) afirmam que usam a Unidade Básica de Saúde (UBS) de referência, mas apenas dois terços responderam que receberam dos profissionais de saúde orientação sobre uso correto de medicações ou acolhimento e classificação de risco. Metade recebeu orientação sobre alimentação saudável, 44\%, sobre atividade física, e pouco mais de um terço, sobre saúde bucal e prevenção de quedas (tabela 1).

A pontuação na escala de autocuidado variou de 51 a 120, com média de 96,4 (d.p. $11,5)$ e mediana de 95 pontos. O ponto de corte para o primeiro quartil foi de 90 pontos, aqui considerado como a medida para o déficit de autocuidado, atingindo $25 \%$ dos idosos e delimitando aqueles que precisam ser alvo de atenção especial da equipe de saúde em virtude das suas características.

Aproveitando para instrumentalizar futuras açóes de saúde, apresentamos na figura 1 os dez itens da escala com maior proporção de respostas negativas - discordo totalmente e discordo -, com destaque para aspectos relacionados à atividade física (39\%), mudanças de hábitos em prol da saúde (20\%), busca de informaçôes sobre efeitos de medicamentos e duração suficiente de sono (16\%).
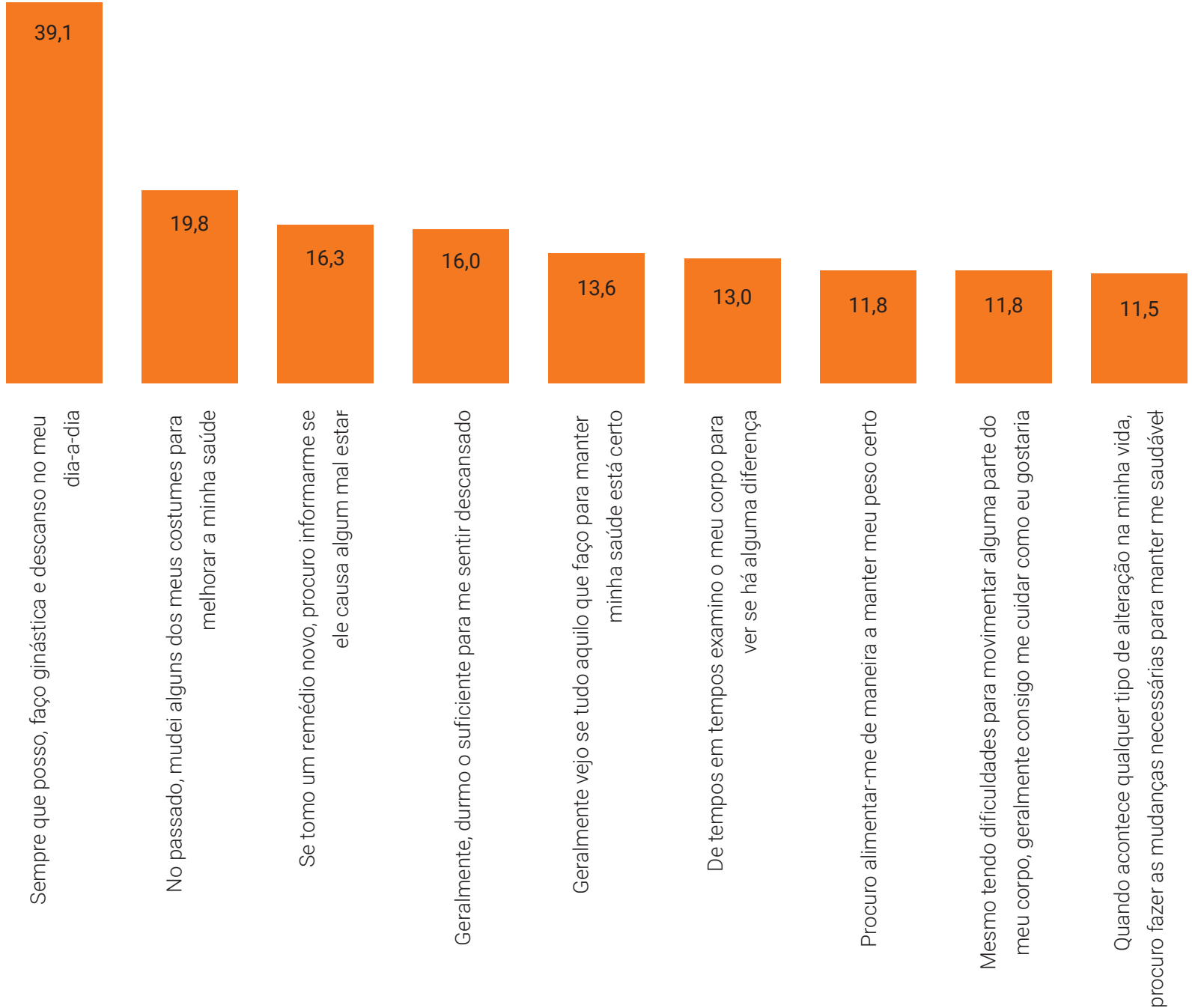

Figura 1. Proporção de idosos que responderam negativamente às afirmações sobre autocuidado. UBS Coral, Lages, SC, 2018. 
(2)

Não foram observadas diferenças no déficit de autocuidado de acordo com o sexo, a idade, a cor da pele, ter companheiro(a) e situação de aposentadoria e trabalho. Quanto mais baixa a escolaridade $(\mathrm{p}=0,026)$ e a renda familiar $(\mathrm{p}=0,073)$, maiores as prevalências do déficit de autocuidado (tabela 1 ).

Idosos classificados como dependentes para atividades básicas da vida diária e os inativos fisicamente apresentaram significativamente mais déficit do que os classificados como independentes $(\mathrm{p}=0,006)$ e como ativos $(\mathrm{p}<0,001)$. Encontrou-se uma prevalência 1,62 vezes maior (IC95\% 1,04-2,51) de déficit de autocuidado entre os indivíduos que não consomem frutas e hortaliças em cinco ou mais dias da semana e 2,08 maior (IC95\% 1,45-2,98) entre os idosos que referiram consumir regularmente carnes com excesso de gorduras (tabela 2).

Tabela 2. Distribuição da amostra e déficit de autocuidado de acordo com características comportamentais. USF Coral, Lages, SC, 2018.

\begin{tabular}{|c|c|c|c|c|}
\hline Característica & $\mathrm{n}(\%)$ & $\begin{array}{l}\text { Déficit de } \\
\text { autocuidado }\end{array}$ & $\begin{array}{l}\text { Razão de prevalências } \\
\text { (IC95\%) }\end{array}$ & p-valor \\
\hline $\begin{array}{l}\text { Fumo }^{1} \\
\text { Nunca fumou } \\
\text { Ex-fumante } \\
\text { Fumante }\end{array}$ & $\begin{array}{l}173(51,6 \%) \\
116(34,6 \%) \\
46(13,7 \%)\end{array}$ & $\begin{array}{l}25,4 \% \\
22,4 \% \\
28,3 \%\end{array}$ & $\begin{array}{c}1,00 \\
0,88(0,58-1,35) \\
1,11(0,66-1,88)\end{array}$ & 0,592 \\
\hline $\begin{array}{l}\text { Abuso de álcool (homens)² } \\
\text { Não } \\
\text { Sim }\end{array}$ & $\begin{array}{l}93(83,8 \%) \\
18(16,2 \%)\end{array}$ & $\begin{array}{l}29,0 \% \\
33,3 \%\end{array}$ & $\begin{array}{c}1,00 \\
1,15(0,56-2,37)\end{array}$ & 0,933 \\
\hline $\begin{array}{l}\text { Abuso de álcool (mulheres) }{ }^{3} \\
\text { Não } \\
\text { Sim }\end{array}$ & $\begin{array}{l}206(96,7 \%) \\
7(3,3 \%)\end{array}$ & $\begin{array}{l}22,3 \% \\
57,1 \%\end{array}$ & $\begin{array}{c}1,00 \\
2,56(1,28-5,10)\end{array}$ & 0,092 \\
\hline $\begin{array}{l}\text { Cuida de algum familiar } \\
\text { Não } \\
\text { Sim }\end{array}$ & $\begin{array}{c}285(84,6 \%) \\
52(15,4 \%)\end{array}$ & $\begin{array}{l}24,2 \% \\
32,7 \%\end{array}$ & $\begin{array}{c}1,00 \\
1,35(0,87-2,10)\end{array}$ & 0,264 \\
\hline $\begin{array}{l}\text { Atividades básicas }{ }^{4} \\
\text { Dependente } \\
\text { Independente }\end{array}$ & $\begin{array}{c}26(7,9 \%) \\
302(92,1 \%)\end{array}$ & $\begin{array}{l}50,0 \% \\
23,5 \%\end{array}$ & $\begin{array}{c}2,13(1,38-3,29) \\
1,00\end{array}$ & 0,006 \\
\hline $\begin{array}{l}\text { Atividades instrumentais (homens) } \\
\text { Dependente } \\
\text { Independente }\end{array}$ & $\begin{array}{l}20(18,0 \%) \\
91(82,0 \%)\end{array}$ & $\begin{array}{l}45,0 \% \\
25,3 \%\end{array}$ & $\begin{array}{c}1,79(0,98-3.24) \\
1,00\end{array}$ & 0,136 \\
\hline $\begin{array}{l}\text { Atividades instrumentais (mulheres) } \\
\text { Dependente } \\
\text { Independente }\end{array}$ & $\begin{array}{l}60(28,6 \%) \\
150(71,4 \%)\end{array}$ & $\begin{array}{l}31,7 \% \\
20,0 \%\end{array}$ & $\begin{array}{c}1,58(0,97-2,59) \\
1,00\end{array}$ & 0,104 \\
\hline $\begin{array}{l}\text { Funcionalidade familiar }{ }^{6} \\
\text { Boa funcionalidade } \\
\text { Moderada disfunção } \\
\text { Elevada disfunção }\end{array}$ & $\begin{array}{l}294(89,1 \%) \\
16(4,8 \%) \\
20(6,1 \%)\end{array}$ & $\begin{array}{l}23,8 \% \\
25,0 \% \\
40,0 \%\end{array}$ & $\begin{array}{c}1,00 \\
1,05(0,44-2,51) \\
1,68(0,95-2,98)\end{array}$ & 0,131 \\
\hline $\begin{array}{l}\text { Atividade física - Ipaq } \\
\quad \text { Ativo }^{7} \\
\text { Inativo }\end{array}$ & $\begin{array}{l}158(47,0 \%) \\
178(53,0 \%)\end{array}$ & $\begin{array}{l}13,3 \% \\
36,0 \%\end{array}$ & $\begin{array}{c}1,00 \\
2,70(1,74-4,22)\end{array}$ & $<0,001$ \\
\hline
\end{tabular}


(e)

\begin{tabular}{lccc}
\hline Característica & $\mathrm{n}(\%)$ & $\begin{array}{c}\text { Déficit de } \\
\text { autocuidado }\end{array}$ & $\begin{array}{c}\text { Razão de prevalências } \\
\text { (IC95\%) }\end{array}$ \\
\hline Consumo regular de frutas e hortaliças & $116(34,3 \%)$ & $21,0 \%$ & 1,00 \\
$\quad$ Sim & $222(65,7 \%)$ & $33,6 \%$ & $1,60(1,11-2,30)$ \\
$\quad$ Não & & & 0,017 \\
Consumo regular de carne com excesso de gordura & $74(22,3 \%)$ & $41,9 \%$ & $2,08(1,45-2,98)$ \\
$\quad$ Sim & $258(77,7 \%)$ & $20,2 \%$ & 1,00 \\
$\quad$ Não & & & $<, 001$ \\
\end{tabular}

1. Inclui indivíduos que fumaram pelo menos um cigarro por dia nos últimos trinta dias (Barbosa AS, 2014).

2. Inclui indivíduos do sexo masculino que consumiram ao menos cinco doses de bebida em uma única ocasião nos últimos trinta dias ${ }^{21}$.

3. Inclui indivíduos do sexo feminino que consumiram ao menos quatro doses de bebida em uma única ocasião nos últimos trinta dias²1.

4. Escala de Katz ${ }^{22}$.

5. Escala de Lawton ${ }^{23}$

6. APGAR de Família ${ }^{24}$.

7. Inclui idosos suficiente e insuficientemente ativos ${ }^{25}$.

Idosos com percepção ruim ou muito ruim de sua saúde tiveram 3,21 vezes mais déficit de autocuidado do que aqueles com percepção boa ou muito boa (IC95\% 2,055,02). Quando comparados aos idosos com três doenças ou menos, aqueles que referiram mais de três doenças tiveram 3,30 vezes mais déficit (IC95\% 1,58-6,89) (tabela 3).

Tabela 3. Distribuição da amostra e déficit de autocuidado de acordo com características de saúde, acesso e utilização de serviços de saúde. USF Coral, Lages, SC, 2018.

\begin{tabular}{|c|c|c|c|c|}
\hline Característica & $\mathrm{n}(\%)$ & $\begin{array}{c}\text { Déficit de } \\
\text { autocuidado }\end{array}$ & $\begin{array}{l}\text { Razão de prevalências } \\
\text { (IC95\%) }\end{array}$ & $\mathrm{p}$-valor \\
\hline $\begin{array}{l}\text { Percepção de saúde } \\
\text { Muito boa/boa } \\
\text { Regular } \\
\text { Ruim/muito ruim }\end{array}$ & $\begin{array}{c}171(51,0 \%) \\
124(37,0 \%) \\
40(11,9 \%)\end{array}$ & $\begin{array}{l}16,4 \% \\
29,0 \% \\
52,5 \%\end{array}$ & $\begin{array}{c}1,00 \\
1,78(1,15-2,74) \\
3,21(2,05-5,02)\end{array}$ & $<0,001$ \\
\hline $\begin{array}{l}\text { Multimorbidade1 } \\
\text { Não } \\
\text { Sim }\end{array}$ & $\begin{array}{l}76(27,1 \%) \\
204(72,9 \%)\end{array}$ & $\begin{array}{c}9,2 \% \\
30,4 \%\end{array}$ & $\begin{array}{c}1,00 \\
3,30(1,58-6,89)\end{array}$ & $<0,001$ \\
\hline $\begin{array}{l}\text { Precisou do serviço de saúde2 } \\
\text { Não } \\
\text { Sim }\end{array}$ & $\begin{array}{c}83(24,6 \%) \\
255(75,4 \%)\end{array}$ & $\begin{array}{l}21,7 \% \\
26,7 \%\end{array}$ & $\begin{array}{c}1,00 \\
1,23(0,78-1,94)\end{array}$ & 0,447 \\
\hline $\begin{array}{l}\text { Utiliza a USF Coral } \\
\text { Sim } \\
\text { Não }\end{array}$ & $\begin{array}{c}278(82,2 \%) \\
60(17,8 \%)\end{array}$ & $\begin{array}{l}24,5 \% \\
30,0 \%\end{array}$ & $\begin{array}{c}1,00 \\
1,23(0,79-1,90)\end{array}$ & 0,465 \\
\hline $\begin{array}{l}\text { Orientação3 sobre alimentação saudável } \\
\text { Sim } \\
\text { Não }\end{array}$ & $\begin{array}{l}139(50 \%) \\
139(50 \%)\end{array}$ & $\begin{array}{l}23,0 \% \\
25,9 \%\end{array}$ & $\begin{array}{c}1,00 \\
1,13(0,74-1,70)\end{array}$ & 0,676 \\
\hline $\begin{array}{l}\text { Orientação sobre saúde bucal } \\
\text { Sim } \\
\text { Não }\end{array}$ & $\begin{array}{c}94(33,8 \%) \\
184(66,2 \%)\end{array}$ & $\begin{array}{l}18,1 \% \\
27,7 \%\end{array}$ & $\begin{array}{c}1,00 \\
1,53(0,94-2,50)\end{array}$ & 0,105 \\
\hline $\begin{array}{l}\text { Orientação sobre risco de quedas } \\
\text { Sim } \\
\text { Não }\end{array}$ & $\begin{array}{c}97(34,9 \%) \\
181(65,1 \%)\end{array}$ & $\begin{array}{l}27,8 \% \\
22,7 \%\end{array}$ & $\begin{array}{c}1,00 \\
1,23(0,81-1,87)\end{array}$ & 0,417 \\
\hline $\begin{array}{l}\text { Orientação sobre atividade física } \\
\text { Sim } \\
\text { Não }\end{array}$ & $\begin{array}{l}122(43,9 \%) \\
156(56,1 \%)\end{array}$ & $\begin{array}{l}22,1 \% \\
26,3 \%\end{array}$ & $\begin{array}{c}1,00 \\
1,19(0,78-1,81)\end{array}$ & 0,510 \\
\hline
\end{tabular}


(e)

\begin{tabular}{lccc}
\hline Característica & $\mathrm{n}(\%)$ & $\begin{array}{c}\text { Déficit de } \\
\text { autocuidado }\end{array}$ & $\begin{array}{c}\text { Razão de prevalências } \\
\text { (IC95\%) }\end{array}$ \\
\hline Orientação sobre uso correto da medicação & $187(67,3 \%)$ & $25,1 \%$ & $1,10(0,70-1,73)$ \\
$\quad$ Sim & $91(32,7 \%)$ & $23,1 \%$ & 1,00 \\
$\quad$ Não & $172(61,9 \%)$ & $26,7 \%$ & $1,29(0,82-2,01)$ \\
$\quad$ Orientação sobre acolhimento4 & $106(38,1 \%)$ & $20,8 \%$ & 1,00 \\
$\quad$ Sim & & & 0,325 \\
Não &
\end{tabular}

1. Cinco ou mais problemas de saúde referidos.

2. Precisou e procurou serviço de saúde.

3. Orientação fornecida por profissional da USF Coral.

4. Inclui acolhimento e classificação de risco.

\section{Discussão}

Os resultados apontam características comportamentais e da condição de saúde importantes ao grupo de maior exposição, em virtude da maior prevalência do déficit de autocuidado: idosos com maior número de doenças, com pior percepção de sua saúde, dependentes para as atividades básicas da vida diária e inativos do ponto de vista da prática de atividade física regular.

Ainda que a maior limitação do estudo seja o fato de este ter um delineamento transversal, não sendo possível afirmar o que veio antes na vida desses idosos, importa que todos esses fatores parecem vir juntos e associar-se negativamente à capacidade de autocuidado. Da mesma forma, o adoecimento em idosos pode afetar de forma importante sua rotina de vida, fazendo com que se tornem mais dependentes e com menor capacidade de cuidar de si mesmos em todas as suas necessidades ${ }^{22,23}$.

Por outro lado, a prática da atividade física nessa etapa da vida demonstra-se protetora, representando maior mobilidade; estabilidade postural; menor risco de quedas; melhoria da função respiratória; controle da gordura corporal; manutenção da massa muscular e densidade mineral óssea; maior circulação do sangue; e redução da frequência cardíaca e dos índices séricos de colesterol como medidas objetivas de saúde física ${ }^{29}$. Além disso, a proteção conferida pela atividade física pode ir além da saúde física, reduzindo sintomas depressivos ou ansiosos em idosos ${ }^{30}$ e representando também uma sensação de saúde, ligada à independência ${ }^{29}$, destacando a contribuição dessa variável para o desenvolvimento de capacidades de autocuidado ${ }^{31}$.

Um destaque positivo foi a observação de que a prevalência de idosos ativos na população estudada $(47,0 \%)$ supera os $30,1 \%$ dos adultos, com 65 anos ou mais, que praticam atividade física no seu tempo livre, conforme Vigitel $^{21}$.

Quanto aos aspectos relacionados ao consumo de alimentos, a pesquisa também apontou uma superioridade entre o consumo regular de frutas e hortaliças por parte dos indivíduos entrevistados (34,3\%) frente ao consumo registrado nas capitais brasileiras pelo Vigitel $^{21}$ para a mesma faixa etária $(27,8 \%)^{21}$. Esse indicador é apontado pela Organização Mundial de Saúde (OMS) como um importante fator de proteção e de prevenção para as doenças crônicas não transmissíveis (DCNT), desde que atingida a recomendação para um consumo de $400 \mathrm{~g} /$ dia, equivalente a cinco porçóes de frutas, legumes e/ou hortaliças. 
Nesse sentido, há que se pensar em ações intersetoriais com organizações não governamentais (ONGs) ou com as Secretarias de Meio Ambiente que busquem construir hortas comunitárias que aumentem a oferta desses alimentos para populaçóes de menor renda per capita, ao mesmo tempo em que propiciam discussóes sobre o autocuidado, a alimentação saudável e o desestímulo ao uso excessivo de medicamentos ${ }^{32}$.

Entre os determinantes sociais, o estudo encontrou associação significativa entre baixa escolaridade e déficit de autocuidado. As capacidades de envolver-se e de executar açốes para o autocuidado são aprendidas e estão sujeitas a fatores condicionantes como a escolaridade ${ }^{33}$. Indivíduos com menos anos de estudo apresentaram piores desfechos, impondo maior preocupação e atenção com a saúde entre os indivíduos idosos de menor escolaridade.

Ao avaliar a influência da escolaridade na percepção sobre alimentos considerados saudáveis, Moura e Masquio ${ }^{33}$ apontam para a necessidade de se continuar a investir em educação em saúde, uma vez que encontra em indivíduos de menor escolaridade interpretações errôneas sobre conceitos de alimentação saudável, podendo contribuir negativamente para açốes de autocuidado.

Incentivar o uso da internet, ou mesmo incluir nas atividades coletivas momentos de buscas na rede, pode ser uma estratégia eficaz para que as equipes estimulem os indivíduos a manter dietas mais saudáveis, de acordo com Souza ${ }^{34}$. Este estudo identificou que indivíduos idosos usuários de internet têm uma chance 1,48 vez maior de consumir adequadamente frutas e hortaliças (IC 95\% 1,09-2,01) quando comparados àqueles que não têm esse acesso ${ }^{34}$.

Estudos com indivíduos diabéticos de diferentes faixas etárias também apontam que a baixa escolaridade pode dificultar a adesão ao plano terapêutico tanto pela dificuldade do indivíduo para ler e entender a prescrição quanto por sua menor compreensão dos complexos mecanismos da doença e do próprio tratamento ${ }^{35}$. Os autores recomendam que os profissionais de saúde devem utilizar linguagem adequada e buscar meios que facilitem a compreensão das orientações e ações de educação em saúde nessa população ${ }^{36}$.

Destaca-se aqui a importância de investir na escolarização entre os idosos, bem como realizar açôes de educação em saúde com o objetivo de proporcionar o componente de poder do autocuidado. O importante é que os indivíduos consigam entender, interpretar e aplicar as informações sobre saúde para fazer melhores escolhas no autocuidado. Viabilizar projetos de integração intersetorial, principalmente entre as Secretarias Municipais da Saúde e Educação, pode ser uma estratégia tão potente quanto promover ações de educação em saúde, dependendo de cada realidade local. 


\section{Considerações finais}

Os resultados do estudo apontam para a necessidade de sempre buscar qualificar a assistência na ESF, investindo em educação continuada e permanente dos profissionais e incentivando-os a construir ações de educação em saúde que estimulem comportamentos de prevenção, letramento funcional de saúde e iniciativas de autogestão para indivíduos com doenças crônicas.

Além de permitir a identificação das características ligadas ao autocuidado, testemunhando a importância de as equipes investirem no diagnóstico de suas comunidades, buscou-se refletir sobre a possibilidade de promover açóes direcionadas às suas realidades, aproximando o cidadão de políticas públicas e de recursos da comunidade local.

\section{Contribuições dos autores}

Ambas as autoras participaram ativamente de todas as etapas de elaboração do manuscrito.

\section{Agradecimentos}

Certamente, o sucesso desta pesquisa é fruto de um grande trabalho em equipe. Nossos agradecimentos a cada um de vocês: estudantes; colegas de trabalho; Secretaria Municipal de Saúde; corpo discente e docente do ProfSaúde/UFPEL; e, em especial, à orientadora do trabalho, Elaine Tomasi.

\section{Direitos autorais}

Este artigo está licenciado sob a Licença Internacional Creative Commons 4.0, tipo BY (https://creativecommons.org/licenses/by/4.0/deed.pt_BR).

\section{(cc) BY}

\section{Referências}

1. Organização Mundial da Saúde. Envelhecimento ativo: uma política de saúde. Brasília: OPAS; 2005.

2. Instituto Brasileiro de Geografia e Estatística. Censo Demográfico, 2010. Rio de Janeiro: IBGE; 2010.

3. Veras R. Envelhecimento populacional contemporâneo: demandas, desafios e inovaçôes. Rev Saude Publica. 2009; 43(3):548-54.

4. Miranda GMD, Mendes ACG, Silva ALA. O envelhecimento populacional brasileiro : desafios e consequências sociais atuais e futuras. Rev Bras Geriatr Gerontol. 2016; 19(3):507-19. 
5. Santos JAF. Classe social, território e desigualdade de saúde no Brasil. Saude Soc. 2018; 27(2):556-72.

6. Nunes BP, Thumé E, Facchini LA. Multimorbidity in older adults: magnitude and challenges for the Brazilian health system chronic disease epidemiology. BMC Public Health. 2015; 15(1):1-11.

7. Mendes EV. As redes de atenção à saúde. Cienc Saude Colet. 2010; 15(5):2297-305.

8. Mendes EV. O cuidado das condiçôes crônicas na atenção primária à saúde: $\mathrm{O}$ imperativo da consolidação da estratégia da saúde da família. Brasília: OPAS, Conselho Nacional de Secretários de Saúde; 2012.

9. Starfield B. Atenção primária: equilíbrio entre necessidades de saúde, serviços e tecnologia. Brasília: Unesco, Ministério da Saúde; 2002.

10. Starfield B. Is patient-centered care the same as person-focused care? Perm J. 2011; 15(2):63-9.

11. Stewart M, McWilliam CL, Weston WW, Brown JB, McWhinney I, Freeman T. Medicina centrada na pessoa: transformando o método clínico. 3a ed. Porto Alegre: Artmed; 2017.

12. Brasil. Ministério da Saúde. Diretrizes para o cuidado das pessoas idosas no SUS: proposta de modelo de atenção integral In: 30o Congresso Nacional de Secretarias Municipais de Saúde [Internet]; 2014; Serra, ES. Serra: Conasems; 2014 [citado 7 Jul 2020]. Disponível em: http://bvsms.saude.gov.br/bvs/publicacoes/diretrizes_ cuidado_pessoa_idosa_sus.pdf

13. Brasil. Ministério da Saúde. Secretaria de Atenção à Saúde. Departamento de Atenção Básica. Diretrizes para o cuidado das pessoas com doenças crônicas nas redes de atenção à saúde e nas linhas de cuidado prioritárias. Brasília: Ministério da Saúde; 2013.

14. Merhy E, Feuerwecker L. Novo olhar sobre as tecnologias de saúde: uma necessidade contemporânea. In: Mandarino ACS, Gomberg E, organizadores. Leitura de novas tecnologias e saúde. Salvador: Edufba, Edufs; 2009. v. 1, p. 29-56.

15. Prado Solar LA, González Reguera M, Paz Gómez N, Romero Borges K. La teoría Déficit de autocuidado: Dorothea Orem punto de partida para calidad en la atención. Rev Med Electron. 2014; 6(36):835-45.

16. Queirós PJ. Autocuidado: o contributo teórico de Orem para a disciplina e profissão de Enfermagem. Rev Enf Ref. 2014; 4(3):157-64.

17. Silva JV, Domingues EAR. Adaptação cultural e validação da escala para avaliar as capacidades de autocuidado. Arq Cienc Saude. 2017; 24(4):30.

18. Lange I, Urrutia M, Campos C, Gallegos E, Herrera LM, Jaimovich S, et al. Fortalecimiento del autocuidado como estratégia de la Atención Primaria en Salud: la contribución de las instituciones de salud em América Latina. Geneva: OPAS; 2006.

19. Brasil. Ministério da Saúde. Secretaria de Gestão do Trabalho e da Educação na Saúde. Departamento de Gestão da Educação em Saúde. Política Nacional de Educação Permanente em Saúde. Brasília: Ministério da Saúde; 2009.

20. Barsosa AS, Barbosa LS, Rodrigues L, Oliveira KL, Iracema I, Argimon DL. Múltiplas definições de ser fumante e diagnóstico de tabagismo : uma revisão sistemática. Aletheia. 2014; 45:190-201.

21. Brasil. Ministério da Saúde. Vigitel Brasil 2017 Saúde Suplementar: vigilância de fatores de risco e proteção para doenças crônicas por inquérito telefônico. Brasília: Ministério da Saúde; 2018. 
22. Cozzensa M. Incapacidade funcional para atividades básicas e instrumentais da vida diária em idosos. Rev Saude Publica. 2009; 43(5):796-805.

23. Santos RL, Virtuoso JSJ. Confiabilidade da versão brasileira da escala de atividades instrumentais da vida diária. Rev Bras Promoç Saude. 2008; 21(4):290-6.

24. Silva MJ, Victor JF, Mota FRN, Soares ES, Leite BMB, Oliveira ET. Análise das propriedades psicométricas do APGAR de família com idosos do nordeste brasileiro. Esc Anna Nery. 2014; 18(3):527-32.

25. Matsudo S, Araujo T, Matsudo V, Andrade D, Andrade E, Oliveira LC, et al. Questionário internacional de atividade física (IPAQ): estudo de validade e reprodutibilidade no Brasil. Rev Bras Ativ Fisica Saude. 2001; 6(2):5-18.

26. Silva TR, Menezes PR. Autopercepção de saúde: um estudo com idosos de baixa renda de São Paulo. Rev Med. 2007; 86(1):28-38.

27. Nunes BP, Sandro RRB, De Andrade FB, Souza Junior PRB, Lima-Costa MF, Facchini LA. Multimorbidade em indivíduos com 50 anos ou mais de idade : ELSIBrasil. Rev Saude Publica. 2018; 52 Supl 2:10s.

28. Christiansen TB, Lauritsen JM. EpiData - comprehensive data management and basic statistical analysis system. Odense: EpiData Association; 2010.

29. Maciel MG. Atividade física e funcionalidade do idoso. Motriz Rev Educ Fis. 2010; 16(4):1024-32.

30. Lima AMP, Ramos JLS, Bezerra IMP, Rocha RPB, Batista HMT, Pinheiro WR. Depressão em idosos: uma revisão sistemática da literatura. Rev Epidemiol Controle Infecç. 2016; 6(2):1-7.

31. Matsuo RF, Velardi M, Marques F, Luiza M. Saúde e doença : representaçóes de mulheres idosas praticantes de atividade física. Rev Psicol Deport. 2018; 27(4):97-102.

32. Costa CGA, Garcia MT, Ribeiro SM, Salandini MFS, Bógus CM. Hortas comunitárias como atividade promotora de saúde: uma experiência em Unidades Básicas de Saúde. Cienc Saude Colet. 2015; 20(10):3099-110.

33. Moura A, Masquio D. A Influência da escolaridade na percepção sobre alimentos considerados saudáveis. Rev Educ Pop. 2018; 13(1):82-94.

34. Souza BB, Cembranel F, Hallal ALC, d'Orsi E. Consumo de frutas, legumes e verduras e associação com hábitos de vida e estado nutricional: um estudo prospectivo em uma coorte de idosos. Cienc Saude Colet. 2019; 24(4):1463-72.

35. Rodrigues FFL, Santos MA, Teixeira CRS, Gonela JT, Zanetti ML. Relação entre conhecimento, atitude, escolaridade e tempo de doença em indivíduos com diabetes mellitus. Acta Paul Enferm. 2012; 25(2):284-90.

36. Marques MB, Silva MJ, Coutinho JFV, Lopes MVO. Avaliação da competência de idosos diabéticos para o autocuidado. Rev Esc Enferm USP. 2013; 47(2):415-20. 
The objective of this study was to characterize self-care deficit in association with sociodemographic, behavioral and health status characteristics, as well as with factors related to access and utilization of health services. A population-based, cross-sectional survey was carried out with elderly individuals in the catchment area of a Family Health team in the city of Lages, State of Santa Catarina. Self-care deficit was measured by the Appraisal of Self-Care Agency Scale (ASA-A). The majority of the interviewees were female, white-skinned, aged between 60 and 70 years old. The prevalence of the outcome was $25 \%$ and was significantly related to multimorbidity, poor health self-perception, inadequate food intake, low level of schooling, inactivity, and to dependence on others for the accomplishment of activities of daily living. This strengthens the health teams' role of promoting self-care initiatives, enabling the citizen's closer contact with public policies and local community resources.

Keywords: Self-care. Aging. Multimorbidity. Primary Health Care. Family Health Strategy.

El objetivo de este trabajo fue caracterizar el déficit de autocuidado en asociación con características sociodemográficas, comportamentales, de la condición de salud, de acceso y utilización de servicios de salud. Se realizó una encuesta poblacional, transversal, con ancianos adscritos al territorio del equipo de Salud de la Familia en Lages/Estado de Santa Catarina. El déficit de autocuidado se verificó con el Appraisal of Self-Care Agency Scale (ASA-A). Entre los entrevistados, la mayoría eran del sexo femenino, blancos, con edad entre 60 y 70 años. La prevalencia del resultado fue del $25 \%$ y se relacionó significativamente con: multimorbilidad, peor autopercepción de salud, consumo alimenticio inadecuado, baja escolaridad, inactividad y dependencia para las actividades básicas de la vida diaria, reforzando el papel de los equipos de salud para promover iniciativas de autocuidado, aproximando al ciudadano de políticas públicas y de recursos de la comunidad local.

Palabras clave: Autocuidado. Envejecimiento. Multimorbilidad. Atención Primaria de la Salud. Estrategia Salud de la Familia. 